\title{
Surgical Management of Recurrent Infectious Perivisceral Aortitis With Expanding Aortic Stump Pseudoaneurysm
}

Matthew A. Sherman, BA BA ${ }^{1}$; Jordan P. Knepper, MD $^{1}$;

Jonathan L. Eliason, $\mathrm{MD}^{1}$; Dawn M. Coleman, $\mathrm{MD}^{1}$

\section{ABSTRACT}

Here we present a case of recurrent aortic graft infection (AGI) in a 77-year-old male resulting in progressive perivisceral aortitis and aortic stump pseudoaneurysm one year following graft explantation and creation of an infrarenal aortic stump for AGI. Recurrent AGl is a common, yet diverse, disease process that carries substantial morbidity and mortality. Vascular Surgery, University of Michigan Cardiovascular Center, Ann Arbor, MI 48109, USA

\section{Author E-mail List:}

sherma@med.umich.edu, jordankn@med.umich.edu, jonaelia@med.umich.edu, dawnbarn@med.umich.edu

Corresponding Author: Matthew A. Sherman, BA BA (sherma@med.umich.edu)

Conflicts of Interest: The authors have no conflicts of interest to disclose. Following initial medical management with broad-spectrum antibiotics, the patient was treated surgically with visceral debranching off the thoracic aorta and creation of a descending thoracic aortic stump. In this case, rifampin-bonded grafts were

\section{Introduction}

Recurrent infection following the surgical treatment of aortic graft infection (AGI) is common and carries substantial morbidity and

utilized and the diaphragm was used for aortic reinforcement. The extent of the phlegmon prevented extensive source control, requiring long-term parenteral broadspectrum antibiotics including antifungal coverage against Candida albicans.

The incidence of AGl is relatively low. Reinfection after surgical management for $A G l$ is

mortality. Presentation is usually nonspecific, including vague abdominal or back pain, weight loss, and elevated inflammatory markers. ${ }^{1,2}$ Offending organisms 
are most commonly skin flora, especially Staphylococcus species, but may include gram-negative bacteria and, rarely, fungi., ${ }^{3,4}$ Computed tomography (CT) often reveals periaortic fluid and inflammatory tissue, and fused positron emission tomography (PET)/CT has been shown to further define the areas of infection. ${ }^{5}$

Treatment includes both medical management with antibiotics and surgical management. Graft excision with extra-anatomic bypass was previously regarded as the gold standard operation. However, this method is associated with reinfection rates of approximately $20 \%$, and in situ reconstruction has started to be favored over extra-anatomic bypass. ${ }^{6,7}$ Additionally, there is a defined role for conservative-i.e., nonresectional-management, specifically in the setting of patients unfit for surgery or with inaccessible infectious material. ${ }^{4,5}$

Here, we present the case of recurrent aortic infection resulting in severe perivisceral aortitis and aortic stump pseudoaneurysm one year following the conventional open repair of AGI. The surgical approach, which involved visceral debranching with rifampin-soaked Dacron grafts and creation of a thoracic aortic stump, highlights several important concepts in the management of this complicated and variable disease. Consent to present this case report with imaging was obtained from the patient.

\section{Case Presentation}

A 77-year-old male with hypertension, coronary artery disease, hyperlipidemia, and long-standing tobacco abuse developed a secondary aortoduodenal fistula (SADF) with associated gastrointestinal bleeding 6 weeks following open repair of a juxta-renal abdominal aortic aneurysm (AAA) at an outside hospital. This early complication was managed by the index surgical team, and operative reports note resection of the fourth portion of duodenum, creation of a duodenal-jejunostomy, and tightening of the proximal aortic suture line at the site of pseudoaneurysm with omental reinforcement. The patient was discharged to an extended care facility on postoperative day 26. Nine days later, he re-presented to the outside hospital with vague abdominal complaints and failure to thrive. CT imaging was obtained and revealed peri-graft fluid with gas and a retro-duodenal abscess concerning for anastomotic leak.

Approximately 6 weeks following reoperation, he was transferred to an academic tertiary cardiovascular center for definitive management. He underwent extra-anatomic revascularization via axillo-femoral-femoral bypass followed the next day by formal graft explantation, oversewing of the aorta distal to the renal arteries, and omental flap creation for stump reinforcement. The duodenum was also resected and reconstructed with primary reanastomosis. Operative cultures grew multidrug resistant Enterococcus faecium, Enterococcus faecalis, Candida albicans, and Candida glabrata. The patient's course was complicated by an early bile leak that was managed conservatively with bowel rest, nasogastric decompression, broad-spectrum intravenous antibiotics, and percutaneous drainage. He was discharged to an extended care facility on postoperative day 21 after the bile leak sealed and CT imaging confirmed resolving fluid collections. The patient was discharged with 6 weeks of IV antibiotics followed by extended-course suppressive antibiotics and surveillance CT imaging. Of note, this operation for secondary aortoduodenal fistula was included in a larger series published in Annals of Vascular Surgery. ${ }^{8}$

The patient unfortunately did not maintain follow-up with vascular surgery or infectious disease providers due to large travel distances and was nonadherent to the recommended antibiotic plan. One year later, he presented with subacute abdominal and midback pain. CT imaging revealed new perivisceral inflammation and a small aortic stump pseudoaneurysm (Figure 1A). Conservative medical management with broad-spectrum IV antibiotics targeting his previous operative cultures was recommended. His infection progressed despite subsequent adherence to his treatment 
regimen, and he returned 3 weeks later with worsening symptoms. Repeat CT imaging revealed progressive aortitis and an expanding pseudoaneurysm (Figure 1B, C), and he was offered open surgical reconstruction. Of note, preoperative blood cultures grew Candida albicans.

The thoracic and retroperitoneal abdominal aorta was exposed through a thoracoabdominal

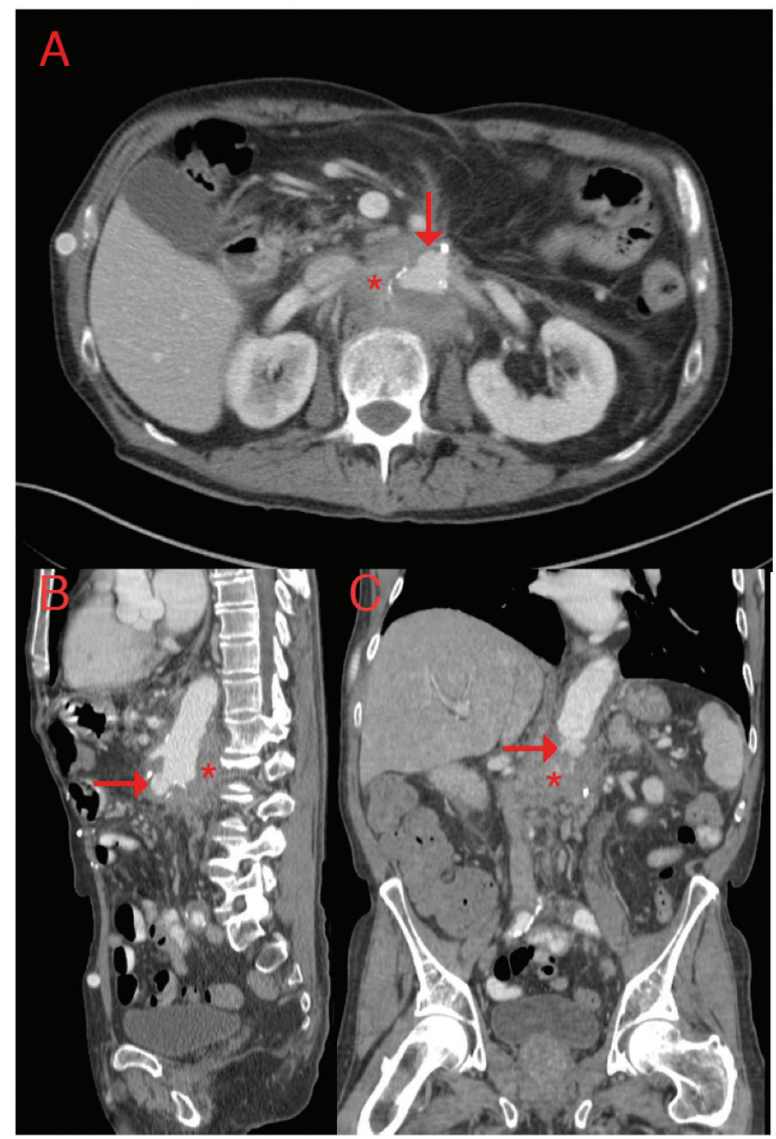

FIGURE 1. Preoperative computed tomography of periaortic phlegmon and aortic stump pseudoaneurysm CT scans of 77-year-old male presenting with subacute abdominal and midback pain one year following management of aortic graft infection with extra-anatomic bypass and graft explantation. Initial cross-sectional CT revealed new perivisceral inflammation surrounding the infrarenal aortic stump (red asterisk) and an early anterior pseudoaneurysm (red arrow) (A). Following 3 weeks of medical management with broad-spectrum intravenous antibiotics, sagittal (B) and coronal (C) CT demonstrated progressive periaortic inflammation (red asterisk) and expanding pseudoaneurysm (red arrow). incision extending through the eighth intercostal space. The thoracic aorta was regular proximal to the diaphragm, but the abdominal aorta and all visceral branches were fully encased by an indurated, adhesive phlegmon. The midthoracic aorta, proximal celiac artery, proximal superior mesenteric artery (SMA), and left midrenal artery were fully exposed for vascular control. Following creation of a longitudinal aortotomy, a proximal anastomosis was created with a bifurcated rifampin-soaked Hemashield graft. End-to-end distal anastomoses to the celiac artery and SMA were constructed sequentially, and the distal left renal artery was debranched with a separate rifampin-soaked Hemashield graft (Atrium, $\mathrm{NH}$ ). To this end, the right renal artery was inaccessible.

Next, the pseudoaneurysm sac was entered. The enclosed material was primarily dense, fibrotic inflammatory tissue with minimal thrombus and no purulence or friable tissue. Finally, the supraceliac aorta was oversewn with 2 separate doublelayered Blalock sutures, and a rifampin-soaked Hemashield "pledget" was used for reinforcement (Figure 2). The crus and diaphragmatic closure provided additional reinforcement to the aortic stump. Surgical drains were not left behind with the exception of a tube thoracostomy. Operative time totaled 604 minutes, and estimated blood loss was 12.5 liters. Operative cultures grew pansensitive Candida albicans.

The immediate postoperative course was complicated by prolonged ventilator support necessitating tracheostomy and by nonoliguric acute renal failure (postoperative creatinine peaked at 2.8 and stabilized at 1.4). He was discharged on postoperative day 22 to a long-term acute care facility for ongoing pulmonary support (ventilator wean). A 6-week course of micafungin, meropenem, and daptomycin was recommended to provide broader coverage specifically against organisms that grew from operative cultures and intra-abdominal fluid from the previous year. Additionally, surveillance CT imaging was requested, 


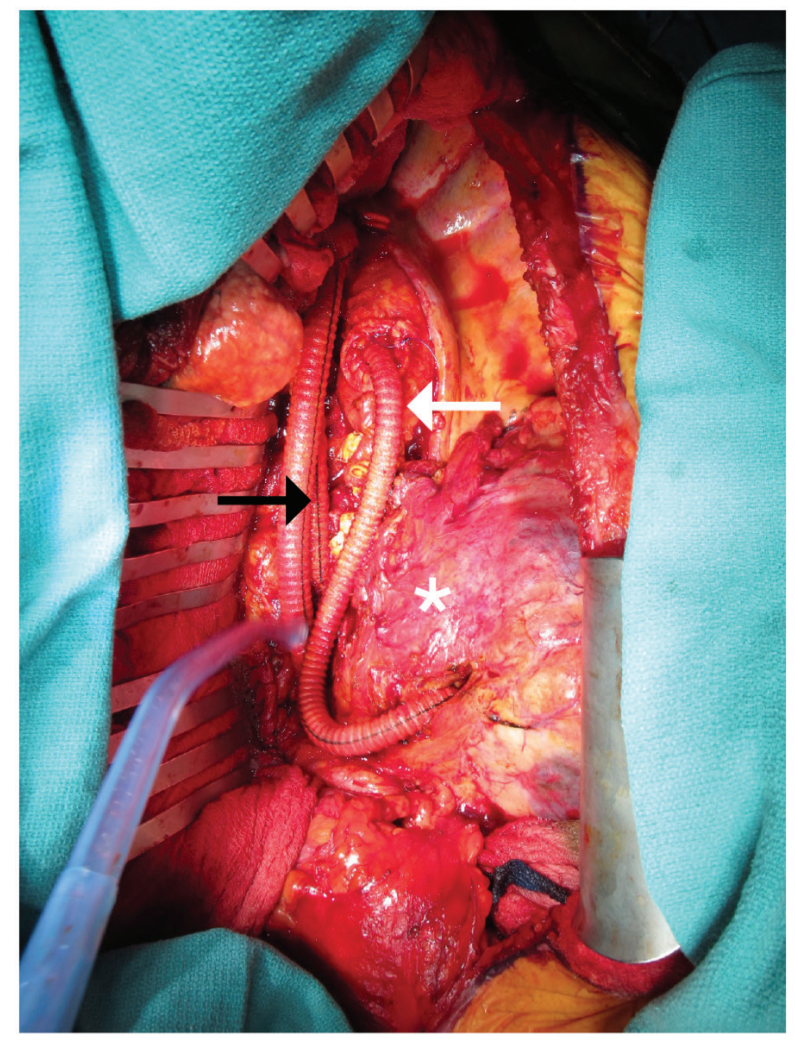

FIGURE 2. Intraoperative photograph demonstrating visceral debranching and creation of a thoracic aortic stump. The celiac and superior mesenteric artery graft limbs are located lateral to the aorta. The aorto-renal bypass is clearly visible (white arrow), and the reinforced thoracic aortic stump (black arrow) lies proximal to this bypass. The phlegmon (white asterisk) encases the perivisceral aorta, left renal artery, and left kidney.

and plans were discussed for indefinite suppressive oral antimicrobial therapy.

Follow-up with vascular surgery was limited due to travel restrictions, but local follow-up with infectious disease was arranged. Interval CT imaging, performed locally at 2 and 6 months, demonstrated stable postsurgical aortic ligation, early occlusion of the celiac graft, and patency of the remaining reconstructions without progressive periaortic inflammation. His compliance with antibiotic therapy remained inconsistent despite multiple clarifications by telephone. Unfortunately, he re-presented 5 months after surgery with acute cholecystitis that was managed with renewed parenteral antibiotics and cholecystostomy tube. He expired 2 months later; cause of death remains poorly defined.

\section{Discussion}

The incidence of AGI ranges from 0.5-5\%.,7 Reinfection following surgical management of AGI occurs at a significantly higher rate than primary AGI, varies based on modality of AGI treatment, and is strongly associated with patient mortality. $3,7,9$ Additionally, aortic stump pseudoaneurysm is a rare but serious complication of aneurysm resection that may result from long-term infection and portend fatal stump blowout. ${ }^{10,11}$ The initial operative approach to AGI aims to treat infection while maintaining distal perfusion. Historically, this has most commonly been executed by total graft excision, local debridement, and extra-anatomic bypass. Another option is graft excision with in situ reconstruction using rifampin-bonded grafts, cryopreserved allografts, or autogenous venous grafts. In situ reconstruction boasts overall improved combined outcomes in terms of amputation, conduit failure, reinfection, and mortality_regardless of the conduit-and has more recently been advocated to supplant extra-anatomic bypass as the first-line treatment for AGI. ${ }^{2}$ Recurrent infection may further complicate AGI and requires a thoughtful, individualized, and often multidisciplinary approach to both medical and complex surgical management. This case specifically highlights several important management principles.

Conforming to recommendations for retroperitoneal exposure in the setting of inflammatory aortic aneurysms, the aorta was exposed through a thoracoabdominal approach limited to the left hemithorax and retroperitoneum. This approach allows for access to the descending thoracic aorta and reduces the chance of visceral injury. ${ }^{2}$ Moreover, this approach avoided a potentially hostile abdomen and adhesions that resulted from the patient's 3 prior laparotomies.

Conduit for visceral debranching proved challenging in this case given the total length required. cryopreserved allograft could have been utilized 
for visceral debranching for this final operation in light of evolving data suggesting lower rates of recurrent infection and aortic blowout. ${ }^{10,11}$ However, there were barriers to urgent acquisition. More importantly, cryopreserved aortoilliac allograft for index aortic reconstruction at the time of SADF could have been considered. Autogenous venous grafts are associated with the lowest rates of reinfection and a favorably low incidence of late graft complications such as stenosis, thrombosis, and dilatation. However, the requirement for preoperative venous duplex ultrasound and longer operative time were limiting. 3,7,10 Rifampin-bonded grafts were ultimately utilized in this case given accessibility. Despite higher rates of reinfection, these grafts have the lowest average rates of adverse events. ${ }^{3,6,7}$ Crucially, recurrent infection is common regardless of conduit, and conduit selection in this case was therefore individualized to the patient and his urgent clinical situation. 9,12

Omental wrapping has been associated with lower rates of reinfection in a certain subset of AGls treated with rifampin-bonded grafts, but options for vascularized coverage were limited in this final case due to the previous creation of an omental flap for aortic stump coverage. ${ }^{13}$ While the use of pericardial or pleural flaps have been described, in this case, the well-vascularized muscle of the diaphragm naturally abutted the terminal thoracic aortic stump at the time of closure and was used as such for reinforcement.

Surgical cultures grew Candida. While fungal infections (usually Candida or Aspergillus) account for a minority of AGIs and most often occur in immunocompromised hosts, the incidence of Candidal infections has been described as high as 38\% in certain series. ${ }^{13,14}$ It remains unclear whether the persistent Candidal infection was simply due to patient nonadherence to the recommended antimicrobial regimen. It is certainly possible that the recurrence and severity of his infectious course were compounded by early bile leak after graft explantation. Perhaps an alternative approach to duodenal management, such as duodenal exclusion with gastro-jejunal bypass, or more aggressive management of the bile leak at the time of identification could have prevented this complication. The resulting densely indurated and inflamed phlegmon precluded the wide local tissue debridement that should be performed for source control. There is, however, a defined role for conservative-i.e., nonresectional-management such as in the setting of serious comorbidity and inaccessible infected tissue, as in this case. Moreover, successful conservative treatment of fungal AGI without surgical intervention has been reported. 4,15,16 Finally, aortic control and suture lines, including the proximal aortic anastomoses and aortic stump, were secured exclusive of the retroperitoneum and phlegmon where the aorta was grossly normal in appearance and isolated to the hemithorax.

\section{Conclusion}

In conclusion, recurrent AGI following explantation for graft infection is well-described and carries the risk of major morbidity and mortality. This case of recurrent perivisceral aortitis associated with aortic stump pseudoaneurysm, which was managed with visceral debranching off the thoracic aorta and creation of a thoracic aortic stump, emphasizes the importance of an individualized surgical approach as well as the imperative for close follow-up and compliance.

\section{References}

1. van Bommel EF, van der Veer SJ, Hendriksz TR, Bleumink GS. Persistent chronic peri-aortitis ("inflammatory aneurysm") after abdominal aortic aneurysm repair: systematic review of the literature. Vasc Med. 2008;13(4):293-303.

2. Todd GJ, and DeRose J J Jr. Retroperitoneal approach for repair of inflammatory aortic aneurysms. Ann Vasc Surg. 1995;9(6):525-534.

3. Perera GB, Fujitani RM, Kubaska SM. Aortic graft infection: update on management and treatment options. Vasc Endovascular Surg. 2006;40(1):1-10. 
4. Lawrence PF. Conservative treatment of aortic graft infection. Semin Vasc Surg. 2011;24(4):199-204.

5. Saleem BR, Meerwaldt R, Tielliu IF, Verhoeven EL, van den Dungen J, Zeebregts CJ. Conservative treatment of vascular prosthetic graft infection is associated with high mortality. Am J Surg. 2010;200(1):47-52.

6. Lew W, Moore W. Antibiotic-impregnated grafts for aortic reconstruction. Semin Vasc Surg. 2011;24(4):211-219.

7. O'Connor S, Andrew P, Batt M, Becquemin JP. A systematic review and meta-analysis of treatments for aortic graft infection. J Vasc Surg. 2006;44(1):38-45.

8. Howard R, Kurz S, Sherman MA, Underhill J, Eliason $\mathrm{J}$, Coleman DM. Contemporary management of secondary aortoduodenal fistula. Ann Vasc Surg. 2015;29(8):1614-1618.

9. Berger P, Van Herwaarden JA, Harkisoen S, De Vries JP, Ekkelenkamp M, Moll FL. Surgical treatment of infected aortic grafts. J Cardiovasc Surg. 2012;53(6):719-734.

10. Dorweiler B, Neufang A, Chaban R, Reinstadler J, Duenschede F, Vahl CF. Use and durability of femoral vein for autologous reconstruction with infection of the aortoiliofemoral axis. J Vasc Surg. 2014;59(3):675-683.

11. Harlander-Locke MP, Harmon LK, Lawrence PF, et al. The use of cryopreserved aortoiliac allograft for aortic reconstruction in the United States. J Vasc Surg. 2014;59(3):669-674.

12. Charlton-Ouw KM, Sandhu HK, Huang G, et al. Reinfection after resection and revascularization of infected infrarenal abdominal aortic grafts. J Vasc surg. 2014;59(3):684-692.

13. Oderich GS, Bower TC, Hofer J, et al. In situ rifampinsoaked grafts with omental coverage and antibiotic suppression are durable with low reinfection rates in patients with aortic graft enteric erosion or fistula. J Vasc Surg. 2011;53(1):99-106, 107 e1-7; discussion 106-107.

14. Bakoyiannis CN, Georgopoulos SE, Tsekouras NS, Klonaris CN, Papalambros EL, Bastounis EA. Fungal infection of aortoiliac endograft: a case report and review of the literature. Ann Vasc Surg. 2007;21(2):228-231.

15. Calligaro KD, Veith FJ, Yuan JG, Gargiulo NJ, Dougherty MJ. Intra-abdominal aortic graft infection: complete or partial graft preservation in patients at very high risk. J Vasc Surg. 2003;38(6):1199-1205.

16. Motloch LJ, Rottlaender D, Darabi T, Joost I, Erdmann E, Hoppe UC. Conservative management of Candida infection of prosthetic aortic graft by means of caspofungin and fluconazole alone. Tex Heart Inst J. 2011;38(2):197-200. 